\title{
Causas de admisión hospitalaria y factores pronósticos en pacientes con vasculitis asociadas a anticuerpos anti-citoplasma de neutrófilo
}

\author{
Sebastián Andrés Muñoz ${ }^{1}$, Ignacio Javier Gandino', María Cristina Basta ${ }^{1}$, Alberto Omar Orden² \\ ${ }^{1}$ Servicio de Clínica Médica. Hospital General de Agudos Juan A. Fernández, ${ }^{2}$ Clínica San Camilo. Ciudad Autónoma de Buenos Aires, Argentina.
}

\begin{abstract}
Resumen
Antecedentes: Existen pocos estudios descriptivos sobre las causas de internación de los pacientes con vasculitis asociadas a ANCA (VAA), todos son retrospectivos y realizados en unidades de cuidados intensivos.

Objetivo: Investigar la epidemiología, los hallazgos clínicos y la evolución de los pacientes con VAA durante su internación, e identificar los factores pronósticos asociados a mortalidad.

Materiales y métodos: Se incluyeron los pacientes con diagnóstico de VAA internados en el Hospital Juan A. Fernández y la Clínica San Camilo (Ciudad Autónoma de Buenos Aires, Argentina), entre el 1 de enero de 2011 y el 31 de diciembre de 2013.
\end{abstract}

Resultados: Treinta y cuatro pacientes fueron incluidos, 18 (53\%) de sexo femenino. Edad media de $60 \pm 12$ años. En 9 (26\%) pacientes el diagnóstico de VAA se realizó en la internación. La Poliangeítis Microscópica (MPA) fue la vasculitis más común (50\%). Dieciocho (53\%) pacientes se internaron por actividad clínica de la vasculitis. Órganos/sistemas afectados: pulmón $(n=9)$, riñón $(n=6)$, otorrinolaringológicas $(n=5)$, sistema nervioso periférico $(n=5)$ y piel $(n=2)$. 0 cho $(23,5 \%)$ pacientes fueron admitidos por complicaciones infecciosas y ocho por otras causas. Fallecieron $8(23,5 \%)$ pacientes, 3 debido a actividad de la vasculitis, 4 debido a complicaciones infecciosas y 1 por falla multiorgánica ( $2^{0}$ infusión pamidronato). Los que ingresaron a UCl tuvieron mayor mortalidad $(p=0,001)$; el sexo $(p=0,69)$, la edad $(p=0,15)$, el diagnóstico "de novo" de vasculitis $(p=0,4)$, el BVAS y VDI no mostraron diferencias entre los sobrevivientes y fallecidos. La mortalidad de los pacientes que ingresaron por actividad de la vasculitis comparado con los que ingresaron por complicaciones infecciosas fue similar $(p=0,6)$.

Conclusiones: La causa más frecuente de internación en pacientes con VAA fue la actividad de la enfermedad, seguida por las causas infecciosas. La mortalidad fue elevada y su principal causa fueron las infecciones independientemente del diagnóstico al ingreso.

Palabras clave: vasculitis, anticuerpos anti-citoplasma de neutrófilo, admisión hospitalaria.

\section{Abstract}

Background: Few retrospective studies have described the clinical course of patients with ANCA-asocciated vasculitis (AAV) admitted to the hospital, all of them in intensive care units (ICU).

Objective: To study the epidemiology, clinical features and outcome of patients with AAV admitted to the hospital, and to identify the prognostic factors associated with mortality.

Methods: Patients with AAV admitted to the Juan A. Fernández Hospital and San Camilo Clinic (Buenos Aires City, Argentina) between January 2011 and December 2013 were included.

Results: Thirty four patients [18 (53\%) female] with an average $60 \pm$ 12 years old were included. AAV was diagnosed in $9(26 \%)$ patients in the hospital. Microscopic Polyangiitis was the most common AAV. Eighteen (53\%) patients were admitted due to active vasculitis. Lung $(n=9)$, kidney $(n=6)$, ear-nose-throat $(n=5)$, peripheral nervous system $(n=5)$ and skin $(n=2)$ were the organs/systems involved. Other reasons for admission were: infection and metabolic conditions [8 (23.5\%) patients each]. Eight (23.5\%) patients died, 3 due to active vasculitis, 4 due to infection and 1 patient due to multiorgan failure after pamidronate treatment. Mortality was significantly higher for patients who were admitted in ICU $(p=0.001)$; gender $(p=0.69)$, age $(p=0.15)$, new diagnosis of AAV $(p=0.4), B V A S$ and VDI showed no significant differences between survival and dead patients. The mortality was similar $(p=0.6)$ between the patients with active vasculitis and the patients with infections.

Conclusion: The main reason for hospitalization in AAV patients was active vasculitis followed by infection. Mortality rate was high and the main causes were infections regardless the diagnosis at admission.

Key words: vasculitis, antineutrophil cytoplasmic antibody, admitted to the hospital. 


\section{Introducción}

Las vasculitis asociadas a ANCA son vasculitis necrotizantes que afectan predominantemente vasos sanguíneos pequeños (arterias pequeñas, arteriolas, capilares y vénulas), se caracterizan por presentar escasos o nulos depósitos inmunes en los tejidos y por la presencia de ANCA con especificidad para las enzimas mieloperoxidasa (MPO) y proteinasa 3 (PR3). La Poliangeítis con Granulomatosis (GPA-Granulomatosis de Wegener), la Poliangeítis Microscópica (MPA) y la Poliangeítis con Granulomatosis Eosinofílica (EGPA-Síndrome de Churg-Strauss) pertenecen a este grupo'.

La presentación clínica, la evolución y el pronóstico de estas enfermedades es muy heterogéneo; algunos pacientes presentan una enfermedad leve, de manejo ambulatoria, mientras que otros presentan una enfermedad grave que supone un riesgo de vida, necesitando su internación para un adecuado manejo.

Los tratamientos actuales para las VAA son muy eficaces; la remisión de la enfermedad, aún en los casos más graves, se logra en un elevado porcentaje de pacientes con la utilización de dosis elevadas de esteroides, ciclofosfamida o rituximab y el uso adecuado del recambio plasmático ${ }^{2,3}$. Sin embargo, el tratamiento inmunosupresor no está exento de complicaciones a corto y largo plazo, muchas de las cuales suelen ser de índole infecciosa ${ }^{4,5}$. Este hecho condujo en los últimos años al desarrollo de nuevas estrategias terapéuticas, y fármacos con similar eficacia a los ya existentes pero con un mejor perfil de seguridad ${ }^{6,7}$.

En la literatura existen pocos estudios descriptivos sobre las causas de internación de los pacientes con VAA; todos son retrospectivos y realizados dentro del ámbito de unidades de cuidados intensivos (UCI). A pesar de ciertas diferencias, coinciden en que la actividad de la vasculitis es la principal causa de internación, seguida de las infecciosas, y que el pronóstico de las VAA continúa siendo pobre con una mortalidad elevada ${ }^{8-11}$.

El propósito de nuestro trabajo fue investigar, en forma prospectiva, la epidemiología, los hallazgos clínicos, la evolución de los pacientes con VAA durante su internación, e identificar los factores pronósticos asociados a mortalidad.

\section{Pacientes y métodos}

\section{Pacientes}

Se incluyeron en forma consecutiva todos los pacientes admitidos a los diferentes servicios de internación del Hospital General de Agudos Juan A. Fernández y la Clínica San Camilo, ambos pertenecientes a la Ciudad Autónoma de Buenos Aires (Argentina), durante el período comprendido entre el 1 de enero de 2011 y el 31 de diciembre de 2013, con diagnóstico previo o "de novo" de VAA.

Los diagnósticos de GPA y EGPA fueron establecidos de acuerdo a los criterios clasificatorios del Colegio Americano de Reumatología ${ }^{12}$, mientras que para el diagnóstico de MPA se utilizó la definición del Consenso de Chapel Hill del año $1994^{13}$.

\section{Métodos}

Las causas de internación fueron divididas en: 1) actividad clínica de la vasculitis, definida como toda aquella manifestación clínica atribuida directamente a la vasculitis; 2) complicaciones infecciosas, confirmada por aislamiento microbiológico o en su defecto por una elevada probabilidad clínica; y 3) otras causas, donde se incluyeron todas las situaciones clínicas no relacionadas a la actividad de la vasculitis o a las complicaciones infecciosas.

En todos los casos de internaciones con más de una causa (ejemplo: neumonía y vasculitis leucocitoclástica cutánea), se consideró como diagnóstico de admisión al problema médico que necesariamente requirió de su manejo y/o tratamiento en la internación. Cuando no se pudo establecer claramente o había más de una causa que necesitaba internación se consideraron ambos en el diagnóstico de ingreso hospitalario.

Lugar de internación: se consideró al sitio inicial de ingreso al hospital (sala de internación general o UCI), debiendo permanecer allí al menos por 48 horas.

Para la evaluación de la severidad y extensión de la enfermedad utilizamos las definiciones del grupo Europeo de Estudio de las Vasculitis (European Vasculitis StudyEUVAS) ${ }^{14}$. Localizada: enfermedad que afecta el tracto respiratorio superior y/o inferior sin afección sistémica ni síntomas constitucionales. Sistémica temprana: enfermedad sin riesgo de pérdida de un órgano vital o de la vida. Generalizada: enfermedad que compromete riñón u otro órgano vital, con creatinina $<5,6 \mathrm{mg} / \mathrm{dl}$. Severa: enfermedad con compromiso renal $\mathrm{u}$ otro órgano vital, con creatinina $>5,6 \mathrm{mg} / \mathrm{dl}$. 
Para determinar actividad de la enfermedad se utilizó el Birmingham Vasculitis Activity Score (BVAS versión 3) y para cuantificar el daño acumulado el Vasculitis Damage Index (VDI) $)^{15,16}$.

\section{Análisis estadístico}

Las variables cualitativas se presentan como $\mathrm{n}$ y porcentaje y las cuantitativas como media y desvío estándar o mediana y rango. Se determinaron los intervalos de confianza 95\% (IC 95\%). Las variables cualitativas fueron comparadas utilizando el chi-cuadrado $\left(\chi^{2}\right)$, o cuando fuera apropiado, la prueba exacta de Fisher. Las variables cuantitativas fueron comparadas utilizando la prueba de t-student y cuando fuera apropiada la prueba de KruskalWallis (para variables no paramétricas). Para todas las pruebas estadísticas utilizadas se consideró como significativa una $\mathrm{p}<0,05$.

\section{Resultados}

Treinta y cuatro pacientes con VAA fueron incluidos para el análisis, 18 (53\%; IC 95\%=30-76\%) de sexo femenino y $16(47 \%$; IC $95 \%=23-71 \%)$ de sexo masculino. La edad media fue de $60 \pm 12$ años (IC 95\%=56-64 años). En 9 (26\%) pacientes el diagnóstico de VAA se realizó en la internación. La MPA fue la vasculitis más común [17

\begin{tabular}{|l|c|}
\hline Característica & $\mathbf{n = 3 4}$ \\
\hline Sexo, $\mathrm{n}(\%)$ & $18(53)$ \\
\hline Femenino & $60 \pm 12$ \\
\hline Edad (años), media \pm desvío estándar & $10(1-36)$ \\
\hline Días de internación, mediana (rango) & $9(26)$ \\
\hline Diagnóstico internación VAA, $\mathrm{n}(\%)$ & \\
\hline Tipo de vasculitis, $\mathrm{n}(\%)$ & $17(50)$ \\
\hline MPA & $7(20)$ \\
\hline GPA & $10(30)$ \\
\hline EGPA & \\
\hline Severidad de VAA, $\mathrm{n}(\%)$ & 0 \\
\hline Localizada & $6(17,6)$ \\
\hline Sistémica temprana & $20(58,8)$ \\
\hline Generalizada & $8(23,5)$ \\
\hline Severa & $10 \pm 9,1$ \\
\hline BVAS, media \pm desvío estándar & $2,5(0-12)$ \\
\hline VDI, mediana (rango) & \\
\hline $\begin{array}{l}\text { VAA: vasculitis asociadas a ANCA; PAM: poliangeítis microscópica; GPA: poliangeítis con } \\
\text { granulomatosis; } \text { EGPA: poliangeítis con granulomatosis eosinofilica; BVAS: Birmingham } \\
\text { vasculitis activity score; VDI: vasculitis damage index. }\end{array}$ \\
\hline
\end{tabular}

Tabla 1. Características generales de los pacientes con VAA.

\begin{tabular}{|l|c|}
\hline Órganos $\mathbf{0}$ sistemas comprometidos & $\mathbf{n}$ \\
\hline Pulmón y vías aéreas inferiores, $\mathrm{n}(\%)$ & $9(50)$ \\
\hline Hemorragia alveolar & 4 \\
\hline Infiltrados pulmonares & 2 \\
\hline Nódulos pulmonares (cavitados) & 2 \\
\hline Estenosis bronquial & 1 \\
\hline Riñón, $\mathrm{n}$ (\%) & $6(33,3)$ \\
\hline Glomerulonefritis paucinmune & 6 \\
\hline Sistema nervioso periférico, $\mathrm{n}(\%)$ & $5(27,7)$ \\
\hline Mononeuritis múltiple & 5 \\
\hline ORL, $\mathrm{n}$ (\%) & $5(27,7)$ \\
\hline Sinusitis & 3 \\
\hline Epistaxis & 1 \\
\hline Hipoacusia & 1 \\
\hline Piel, $\mathrm{n}(\%)$ & $2(11,1)$ \\
\hline Púrpura palpable & 1 \\
\hline Úlcera cutánea & 1 \\
\hline VAA: vasculitis asociadas a ANCA; ORL: otorrinolaringológicas. \\
\hline
\end{tabular}

Tabla 2. Manifestaciones clínicas de los 18 pacientes con VAA que ingresaron por actividad de las vasculitis agrupadas por órganos o sistemas.

pacientes $(50 \%)]$. Treinta $(88 \%)$ pacientes ingresaron inicialmente a la sala de cuidados generales. La mediana de internación fue 10 días (rango=1-36) (Tabla 1).

Dieciocho (53\%; IC 95\%=30-76\%) pacientes se internaron por actividad clínica de la vasculitis. Los órganos o sistemas comprometidos fueron: pulmón $(\mathrm{n}=9)$, riñón $(n=6)$, otorrinolaringológicas $(n=5)$, sistema nervioso periférico $(n=5)$ y piel $(n=2)$. Tres pacientes ingresaron por síndrome pulmo-renal. Las manifestaciones clínicas se detallan en la Tabla 2.

Ocho $(23,5 \%)$ pacientes fueron admitidos por complicaciones infecciosas, 5 infecciones urinarias, 2 neumonías (1 por Streptococcus pneumoniae) y 1 sepsis por Staphylococcus aureus.

Ocho $(23,5 \%)$ pacientes ingresaron por otras causas, 3 fueron debidos a eventos adversos farmacológicos (psicosis esteroidea, aplasia medular por metotrexato y mucositis severa por metotrexato), 2 debidos a infusión de fármacos, y el resto a isquemia intestinal por hernia atascada, hiperglucemia y aplastamiento vertebral por osteoporosis.

Fallecieron $8(23,5 \%)$ pacientes, 3 debido a actividad de la vasculitis ( 2 pacientes con síndrome pulmo-renal y 1 por insuficiencia renal terminal), 4 debido a complicaciones infecciosas (las causas fueron sepsis $2^{\circ}$ perforación 
intestinal, sepsis generalizada, infección urinaria complicada, neumonía intra-hospitalaria por Acynetobacter Baumannii) y 1 paciente falleció poco después de la infusión de pamidronato con un cuadro clínico de falla multiorgánica.

\begin{tabular}{|l|c|c|c|}
\hline & \multicolumn{2}{c|}{ Resultado } & \\
\hline \multicolumn{1}{|c|}{ Variables } & Vivos & Fallecidos & p \\
\hline Todos los pacientes & \multicolumn{3}{|c|}{} \\
\hline BVAS & $9(8,6)$ & $13(10,2)$ & 0,22 \\
\hline VDI & $2(0-12)$ & $3(0-12)$ & 0,3 \\
\hline Pacientes actividad de vasculitis & \multicolumn{3}{|c|}{} \\
\hline BVAS & $15,9(5,2)$ & $16(7,8)$ & 0,91 \\
\hline VDI & $0,5(0-12)$ & $4(0-12)$ & 0,22 \\
\hline BVAS: Birmingham vasculitis activity score; VDI: Vasculitis damage index. \\
\hline
\end{tabular}

Tabla 3. Comparación del BVAS y el VDI según sobrevida.

El análisis de los factores pronósticos mostró que los pacientes que ingresaron a UCI tuvieron mayor mortalidad respecto a los que ingresaron a la sala de cuidados generales ( $100 \%$ vs. $13,3 \%$; $\mathrm{p}=0,001)$, sin embargo el sexo $(p=0,69)$, la edad $(p=0,15)$, el diagnóstico "de novo" de vasculitis $(\mathrm{p}=0,4)$ y los índices de actividad y daño no mostraron diferencias estadísticamente significativas entre los sobrevivientes y fallecidos (Tabla 3). La mortalidad de los pacientes que ingresaron por actividad de la vasculitis fue similar a la que presentaron los pacientes que ingresaron por complicaciones infecciosas ( $22 \%$ vs. $25 \%$; $p=0,6)$.

\section{Discusión}

El objetivo de nuestro estudio fue describir la epidemiología, los hallazgos clínicos y el pronóstico de los pacientes con VAA que fueron internados en la sala de cuidados generales y en UCI.

Los pacientes con VAA suelen presentar manifestaciones clínicas graves o complicaciones del tratamiento inmunosupresor, principalmente de etiología infecciosa, que justifican frecuentemente su internación ${ }^{8-11}$.

En el presente estudio, la causa más frecuente de ingreso hospitalario fue la actividad clínica de la vasculitis $(53 \%)$, seguido por las causas infecciosas $(23,5 \%)$. La cuarta parte de los pacientes fueron diagnosticados de VAA en la internación (Tabla 4). Los órganos más afectados fueron el pulmón, el riñón y el sistema nervioso periférico en sus formas más graves (hemorragia alveolar, glomerulonefritis, mononeuritis múltiple), mientras que las manifestaciones otorrinolaringológicas, aunque frecuentes, no fueron razones directas de internación. Esto es especialmente importante para el médico internista que debe incluir dentro de los diagnósticos diferenciales a las VAA en pacientes internados con manifestaciones clínicas

\begin{tabular}{|c|c|c|c|c|c|}
\hline Características & $\begin{array}{c}\text { Cruz (8) (Francia) } \\
2003 n=26\end{array}$ & $\begin{array}{c}\text { Khan (9) (MN,USA) } \\
2007 n=38\end{array}$ & $\begin{array}{c}\text { Frausova (10) } \\
\text { (Rep. Checa) } \\
2008 n=30\end{array}$ & $\begin{array}{c}\text { Befort (11) } \\
\text { (Francia) } \\
2013 n=31\end{array}$ & $\begin{array}{c}\text { Muñoz } \\
\text { (Argentina) } \\
2014 n=34\end{array}$ \\
\hline \multicolumn{6}{|l|}{ Tipo de vasculitis } \\
\hline MPA & $4(15,3)$ & $16(42)$ & $10(33,3)$ & $2(6,4)$ & $17(50)$ \\
\hline EGPA & $7(26,9)$ & $1(3)$ & - & $1(3,2)$ & $10(30)$ \\
\hline GPA & $5(19,2)$ & $19(50)$ & $20(66,7)$ & $15(48,4)$ & $7(20)$ \\
\hline Otras & $10(38,4)$ & $2(5)$ & - & $13(41,9)$ & - \\
\hline Diagnóstico "de novo" & $11(42,3)$ & $12(31,5)$ & $12(40)$ & $7(23)$ & $9(26)$ \\
\hline \multicolumn{6}{|l|}{ Causas de internación } \\
\hline Actividad vasculitis & $20(79,9)$ & $18(47,3)$ & $13(43,3)$ & $19(61)$ & $18(53)$ \\
\hline Infecciones & $3(11,5)$ & $7(18,4)$ & $7(23,3)$ & $8(26)$ & $8(23,5)$ \\
\hline Otras & $3(11,5)$ & $13(34,2)$ & $10(33,2)$ & $4(13)$ & $8(23,5)$ \\
\hline Mortalidad & $4(15)$ & $4(11)$ & $10(33,3)$ & $16(52)$ & $8(23,5)$ \\
\hline \multicolumn{6}{|l|}{ Causas de muerte } \\
\hline Infecciones & $3(75)$ & $4(100)$ & $5(50)$ & $10(62,5)$ & $4(50)$ \\
\hline Actividad vasculitis & $1(25)$ & - & $2(20)$ & $4(25)$ & $3(37,5)$ \\
\hline Otras & - & - & $3(30)$ & $2(12,5)$ & $1(12,5)$ \\
\hline
\end{tabular}

Tabla 4. Tabla comparativa de las principales características entre las series publicadas. 
no explicadas por causas habituales, sobre todo en aquellas que involucran al pulmón, al riñón y al SNP.

La MPA fue la VAA más frecuente (50\%), seguida por la EGPA (30\%) y en tercer lugar GPA (20\%). Esta frecuencia de distribución, aunque posiblemente casual, difiere de las otras series donde la GPA es la VAA más frecuente (Tabla 4). Este hecho podría deberse a la diferencia geográfica de prevalencia descripta para la GPA y la MPA. Tanto en el hemisferio norte ${ }^{17-19}$ como en el hemisferio $\operatorname{sur}^{20}$, se observó que la prevalencia de la GPA tiende a incrementarse hacia los polos, mientras que lo contrario sucede con la MPA, cuya prevalencia es mayor hacia el ecuador; esta diferencia geográfica de prevalencia no se observó para la EGPA. Buenos Aires se encuentra en el hemisferio sur en una latitud $\left(34^{\circ} \mathrm{S}\right)$ más próxima al ecuador que el resto de las ciudades [Rochester, MN, USA ${ }^{8}$, París y Montpellier, Francia ${ }^{9,11}$ y Praga, República Che$\mathrm{Ca}^{10}$, todas del hemisferio norte] desde las cuales provienen las series publicadas.

La mortalidad intrahospitalaria en nuestra serie fue del $23 \%$. Este porcentaje es ligeramente inferior al observado en otras series que incluyeron pacientes con enfermedades reumáticas sistémicas internados en UCI $(25-50 \%)^{21,22}$, pero se asemeja más a la mortalidad descripta en las series que incluyeron específicamente pacientes con vasculitis sistémicas $(11-52 \%)^{8-11}$. Sin embargo, la interpretación de estos datos debe hacerse con precaución porque en nuestra serie los pacientes fueron internados, en un elevado porcentaje, en la sala de cuidados generales lo que presupone una menor gravedad clínica comparado con las demás series.

Las causas de muerte fueron las infecciones en el 50\% (4/8) de los pacientes y en el 37,5\% (3/8) la actividad de la vasculitis. Las infecciones en general son una de las principales causa de mortalidad hospitalaria ${ }^{23-27}$. Nuestros resultados no difieren de los reportados en las series de Cruz y cols. ${ }^{8}$, Khan y cols. ${ }^{9}$, Frausova y cols. ${ }^{10}$ y Befort y cols. ${ }^{11}$, donde la causa principal de muerte fueron las infecciones, independientemente de la causa inicial de internación, seguida por la actividad de la vasculitis (Tabla 4). El compromiso renal estuvo presente en todos nuestros pacientes que fallecieron por actividad de la vasculitis. La insuficiencia renal aguda es un conocido factor de riesgo independiente de mortalidad en pacientes ingresados a $\mathrm{UCI}^{28}$, y también es un factor de mal pronóstico bien establecido en los pacientes con VAA ${ }^{29}$. Mukhtyar y cols. ${ }^{30}$ realizaron una revisión sistemática de los estudios sobre VAA con el objetivo de identificar factores que afecten la remisión, recaída, función renal y sobrevida. El compromiso renal $(\mathrm{HR}=4,45$, IC 95\%=1,48-13,65), el deterioro de la función renal $(H R=5,10$, IC $95 \%=1,59-10,16)$ y la necesidad de diálisis al momento del diagnóstico $(\mathrm{HR}=8,2$, IC $95 \%=2,03-33,11)$, entre otros factores, influyeron negativamente en la sobrevida de los pacientes.

La mortalidad del grupo de pacientes que ingresó por actividad de la vasculitis fue similar a la del grupo que ingresó por complicaciones infecciosas $(22 \%$ vs. $25 \%$; $\mathrm{p}=0,6)$. Cruz y cols. ${ }^{9}$ en su serie encontró una tendencia $(67 \%$ vs. $9 \% ; p=0,05)$ a una mayor mortalidad en el grupo de pacientes que ingresó a UCI por infecciones. Frausova y cols. ${ }^{10}$ también observó una elevada mortalidad en el grupo que se internó por complicaciones infecciones (5/7 pacientes; $71 \%$ ) comparado con el grupo de pacientes internado por actividad de la vasculitis. La interpretación de estas observaciones resulta dificultosa; por un lado el número de pacientes en las diferentes series es muy pequeño, y por el otro, la existencia de múltiples factores no analizados que posiblemente hubieran modificado los resultados finales.

No encontramos relación entre la actividad de la vasculitis determinada por el BVAS y mortalidad intrahospitalaria. Nuestra observación concuerda con los resultados hallados en las series previas ${ }^{8-11}$. En nuestro trabajo la determinación del BVAS fue prospectiva, esto nos diferencia de las demás series donde el índice fue valorado retrospectivamente; en sentido estricto el BVAS fue evaluado y validado para su uso en forma prospectiva ${ }^{15}$. La correcta determinación del BVAS es importante porque minimiza el error de medición de la actividad de la enfermedad, y su impacto en el análisis final de los resultados. Por otra parte, en todas las series publicadas ${ }^{8-11}$ y en la nuestra en particular, la actividad de la vasculitis como causa de muerte fue poco frecuente. Este hecho pudo contribuir a la ausencia de correlación entre el BVAS y mortalidad. Por último, el BVAS es un sistema de puntuación validado para determinar actividad y no para predecir el pronóstico en pacientes con VAA ${ }^{15,31}$.

No observamos relación entre el daño acumulado determinado por VDI y mortalidad intrahospitalaria. El VDI es una medida del impacto causado por la enfermedad y su tratamiento, validada para su uso desde el comienzo de la enfermedad ${ }^{16}$. La aplicación regular del VDI a los 
pacientes con vasculitis demostró que la acumulación del daño ocurre frecuentemente en estadios tempranos de la enfermedad, y tiene gran impacto en la mortalidad a largo plazo $^{32}$. Sería de esperar que pacientes con enfermedad más grave tengan un mayor daño acumulado, y posiblemente ante una complicación aguda sean más propensos a un desenlace fatal. Sin embargo, esta hipótesis no pudo ser comprobada por nosotros.

Limitaciones de nuestro estudio: al ser dos centros de referencia en VAA, no podemos obviar el sesgo de selección de la muestra que pudiera haber modificado los resultados; por otra parte el número de pacientes incluidos para el análisis fue relativamente pequeño, lo que hace difícil la generalización de los hallazgos.

\section{Conclusiones}

En nuestra serie, la causa más frecuente de internación en pacientes con VAA fue la actividad de la enfermedad, seguida por las causas infecciosas. La mortalidad fue elevada y su principal causa fueron las infecciones independientemente del diagnóstico al ingreso. Finalmente, la observación en nuestra serie de una mortalidad significativamente mayor de los pacientes que ingresaron a la unidad de cuidados intensivos (con índices de actividad y daños similares a las otras series) plantea la necesidad de reafirmar lo importante del diagnóstico precoz y de la revisión de los abordajes terapéuticos.

Los autores declaran no tener ningún conflicto de interés al momento de la redacción del artículo.

\section{Bibliografía}

1. Jennette JC, Falk RJ, Bacon PA, et al. 2012 Revised International Chapel Hill Consensus Conference Nomenclature of Vasculitides. Arthritis Rheum 2013; 65(1):1-11.

2. Mukhtyar C, Guillevin L, Cid M, et al. EULAR recommendations for the management of primary small and medium vessel vasculitis. Ann Rheum Dis 2009; 68:310-317.

3. Schönermarck U, Gross WL, de Groot K. Treatment of ANCA-associated vasculitis. Nat Rev Neprhol 2013; Nov 5. doi: 10.1038/nrneph.2013.225. [Epub ahead of print]

4. Little MA, Nightingale P, Verburgh CA, et al. Early mortality in systemic vasculitis: relative contribution of adverse events and active vasculitis. Ann Rheum Dis 2011; 69:1036-1043.

5. Wall N, Harper L. Complication of long-term therapy for ANCA-associated systemic vasculitis. Nat Rev Nephrol 2012; 8:523-532.

6. Jones RB, Tervaert JW, Hauser T, et al. Rituximab versus cyclophosphamide in ANCA-associated renal vasculitis. N Engl J Med 2010; 363(3):211-220.

7. Stone JH, Merkel PA, Spiera R, et al. Rituximab versus cyclophosphamide for ANCA-associated vasculitis. N Engl J Med 2010; 363(3):221-232.

8. Cruz BA, Ramanoelina J, Mahr A, et al. Prognosis and outcome of 26 patients with systemic necrotizing vasculitis admitted to the intesive care unit. Rheumatology 2003; 42:1183-1188.

9. Khan SA, Subla MR, Behl D, Specks U, Afessa B. Outcome of patients with small-vessel vasculitis admitted to a medical ICU. Chest 2007; 131(4):972-976.

10. Frausova D, Brejnikova M, Hruskova Z, Rihova Z, Tesar V. Outcome of thirty patients with ANCAassociated renal vasculitis admitted to the intensive care unit. Ren Fail 2008; 30(9):890-895.

11. Befort P, Corne P, Filleron T, et al. Prognosis and ICU outcome of systemic vasculitis. BMC Anesthesiology 2013; 13:27-33.

12. Hunder GG, Arend WP, Bloch DA, et al. The American College of Rheumatology 1990 criteria for the classification of vasculitis. Arthritis Rheum 1990; 33:1065-1067.

13. Jennette JC, Falk RJ, Andrassy K, et al. Nomenclature of systemic vasculitides: the proposal of an international consensus conference. Arthritis Rheum 1994; 37:187-192.

14. Hellmich B, Flossmann O, Gross WL, et al. EULAR Recommendations for Conducting Clinical Studies and/or Trials in systemic vasculitis: Focus on ANCA-associated vasculitis. Ann Rheum Dis 2007; 66(5):605-617.

15. Mukhtyar C, Lee R, Brown D, et al. Modification and validation of the Birmingham Vasculitis Activity Score (version 3). Ann Rheum Dis 2009; 68(12):1827-1832.

16. Exley AR, Bacon PA, Luqmani RA, et al. Development and initial validation damage index for the standardized clinical assessment of damage in 
the systemic vasculitides. Arthritis Rheum 1997; 40(2):371-340.

17. Watts RA, Gonzalez-Gay MA, Lane SE, et al. Geoepidemiology of systemic vasculitis: comparison of the incidence in two regions of Europe. Ann Rheum Dis 2001; 60:170-172.

18. Watts RA, Lane SE, Scott DG, et al. Epidemiology of vasculitis in Europe. Ann Rheum Dis 2001; 60:1156-1157.

19. Ntatsaki E, Watts RA, Scott DG. Epidemiology of ANCA-associated vasculitis. Rheum Dis Clin North Am 2010; 36:447-461.

20. Gibson A, Stamp LK, Chapman PT, el al. The epidemiology of Wegener's granulomatosis and microscopic polyangeiitis in a Southern Hemisphere region. Rheumatology (Oxford) 2006; 45:624-628.

21. Semple D, Keogh J, Forni L, Venna R. Clinical review: Vasculitis in the intensive care unit, part 2: Treatment and prognosis. Crit Care 2005; 9:193-197.

22. Kollef MH, Enzenauer RJ. Predicting outcome from intensive care for patients with rheumatologic disease. J Rheumatol 1992; 19:1260-1262.

23. Cervera R, Khamashta M, Font J, et al. Morbidity and mortality in systemic lupus erythematosus during a 5-year period. A multicenter prospective study of 1000 patients. European Working Party on Systemic Lupus Erythematosus. Medicine (Baltimore) 1999; 78:167-175.

24. Godeau B, Mortier E, Roy PM, et al. Short and long-term outcomes for patients with systemic rheumatic diseases admitted to intensive care units: a prognostic study of 181 patients. J Rheumatol 1997; 24:1317-1323.
25. Gallagher H, Kwan JT, Jayne DR. Pulmonary renal syndrome: a 4-year, single-center experience. Am J Kidney Dis 2002; 39:42-47.

26. Jayne DR, Rasmussen N, Andrassy K, et al. A randomized trial of maintenance therapy for vasculitis associated with antineutrophil cytoplasmic autoantibodies. N Eng J Med 2003; 349:36-44.

27. Booth AD, Almond MK, Burns A, et al. Outcome of ANCA-associated renal vasculitis: a 5-year retrospective study. Am J Kidney Dis 2003; 41:776784.

28. Raffin TA. Intensive care unit survival patients with systemic illness. An Rev Respir Dis 1989; 140:S28S35.

29. Rihova Z, Jancova E, Merta M, et al. A long-term outcome of patients antineutrophil cytoplasmic autoantibodies-associated vasculitis with renal disease. Kidney Blood Press Res 2005; 28:144-152.

30. Mukthyar C, Flossmann O, Hellmich B, et al. Outcome from studies of antineutrophil cytoplasm antibody associated vasculitis: a systematic review by the European League Against Rheumatism systematic vasculitis task force. Ann Rheum Dis 2008; 67:1004-1010.

31. Koldingsnes W, Nossent JC. Baseline features and initial treatment as predictors of remission and relapse in Wegener Granulomatosis. J Rheumatol 2003; 30:80-88.

32. Exley A, Carruthers DM, Luqmani RA, et al. Damage occurs early in systemic vasculitis and is an index of outcome. QJM 1997; 90:391-399. 